wait times exceed a reasonable period. Crowding typically involves patients being monitored in non-treatment areas (eg, hallways) awaiting ED treatment beds or inpatient beds. Crowding may also involve an inability to appropriately triage patients, with large numbers of patients in the ED waiting area of any triage assessment category." This issue of ED overcrowding becomes a serious issue, particularly in resource-limited emergency centers.

Methods: An ED overcrowding assessment questionnaire was prepared based on the conceptual model of Emergency Department crowding, and was used to assess particular causes of ED crowding. A well validated NEDOCS (National Emergency Department Overcrowding Score) tracking tool was used to assess NEDCOS level of a particular ED.

Results: Micro-level causes of ED Overcrowding Summary Table. Average NEDCOS score for one month is 141-180/ Severe Overcrowding.

Conclusion: Physical capacity of ED and hospital should be increased. Hospitals should have added express admission units.

\begin{tabular}{|l|c|}
\hline Causes of overcrowding & Percentage \\
\hline $\begin{array}{c}\text { Those patients who need emergency care in the } \\
\text { ED (seriously ill and injured). }\end{array}$ & $1.5 \%$ \\
\hline $\begin{array}{c}\text { Unscheduled urgent care patients in the ED } \\
\text { (patients from follow up clinics with acute } \\
\text { problems). }\end{array}$ & $34 \%$ \\
\hline $\begin{array}{c}\text { Safety net care patients in the ED (patients } \\
\text { having barriers even for unscheduled care). }\end{array}$ & $31 \%$ \\
\hline $\begin{array}{c}\text { Throughput component as a cause of crowding } \\
\text { (includes patient length of stay in the ED as a } \\
\text { potential contributing factor to ED crowding). }\end{array}$ & $33 \%$ \\
\hline $\begin{array}{c}\text { Output component as a cause of ED crowding } \\
\text { (involves disposition of ED patients). }\end{array}$ & \\
\hline
\end{tabular}

Prehosp Disaster Med 2017;32(Suppl. 1):s32-s33

doi:10.1017/\$1049023X17001030

"No Bed Syndrome": Unmet Demand for Hospital Beds in the Emergency Department (ED) of Komfo Anokye Teaching Hospital (KATH)

Joseph Bonney

Emergency Medicine, Komfo Anokye Teaching Hospital, Kumasi/ Ghana

Study/Objective: To determine the trend of bed usages in the Emergency Department of KATH.

Background: The issues of ED bed management are problems of the health sector and to determine if bed usage trends are important for planning and effective management of an ED.

Methods: A quantitative, non-interventional, cross-sectional method was used to collect data on the times for all the major events in the ED, and the number of free beds in the main wards for all patients presenting in a 4-weeks duration.
The data from the time series and mobile application were entered into a Microsoft Excel Module to determine the averages of the demand, discharge, free beds, and admissions in 24 hours.

Results: The results showed an increased demand for beds with multiple peaks: from $0700 \mathrm{hrs}-1200 \mathrm{hrs}, 1500 \mathrm{hrs}$ to $1600 \mathrm{hrs}$, and $1800 \mathrm{hrs}$ to $2000 \mathrm{hrs}$. There are similar times for the discharge and admission to the ward. All values decline from $22 \mathrm{hrs}$ toward 00:00 hrs. Although there are demands from $0000 \mathrm{hrs}$ to $0700 \mathrm{hrs}$, there are virtually no discharges and admissions during these periods. The free beds had a steady rate across all hours of the day, but have no corresponding response to the pattern of admissions to the wards; $r=0.16$ (weak correlation).

Conclusion: The trend of bed usage is important for planning, and can be a tool to determine the number of staff per shift and reduce $\mathrm{ED}$ overcrowding.

Prehosp Disaster Med 2017;32(Suppl. 1):s33

doi:10.1017/S1049023X17001042

Overcrowding in a Low Resource Emergency Setting in

West Africa: Perceptions by Health Workers in the Accident and Emergency Center, Komfo Anokye Teaching Hospital (Kath) Kumasi, Ghana

Nana Serwaa A. Quao, Joseph Bonney, Paa Kobina Forson, George Oduro

Emergency Medicine, Komfo Anokye Teaching Hospital, Kumasi/ Ghana

Study/Objective: To assess the health workers' perception of overcrowding in the Emergency Department (ED).

Background: Emergency Department (ED) overcrowding is gradually becoming a growing trend in many hospitals worldwide. ED overcrowding has negative effects on the quality of patients' care. It increases staff stress and produces poor work satisfaction among health workers. KATH ED receives an average of 84 patients daily with overcrowding and long boarding hours being challenges.

Methods: A cross-sectional study which recruited 110 ED health care workers using systematic random sampling was carried out for three months in 2015. Structured questionnairebased interviews which were pretested and validated were administered. Analysis was done using Epi Info 7 by the CDC. Results: Of the 110 health workers, 59 (53.64\%) were nurses and $51(46.36 \%)$ were doctors. Females were $52(47.27 \%)$ and males were $58(52.73 \%)$. The perceived average waiting time of a patient to see a physician at the ED was 30 minutes. $24.54 \%$ thought the afternoon shifts were congested. Inappropriate referrals (59.63\%) and delays in getting radiological imaging (49.07\%) were the main perceived causes of overcrowding. The negative effects of overcrowding on health workers included increased staff stress (71.03\%), poor work satisfaction (57.41\%), and increased margin of errors (32.41\%). 95\% said that overcrowding in the ED contributed to poor patient outcome by increasing staff exhaustion (40.37\%) and compromising quality of patient care (36.70\%). Health personnel strongly agreed that equipping district hospitals to handle non-emergency cases $(77.78 \%)$, appropriate referral system (75.93\%), and provision of adequate logistics and 
consumables (57.41\%) are ways to curb overcrowding. 98\% agreed that six hourly bed occupancy data from the admitting wards was important to reduce $\mathrm{ED}$ overcrowding.

Conclusion: Overcrowding in the ED is perceived to cause staff burn-out and result in poor patient outcomes. Evidencebased interventions may improve overcrowding in EDs.

Prehosp Disaster Med 2017;32(Suppl. 1):s33-s34

doi:10.1017/S1049023X17001054

Waiting Times in a Tertiary Academic Hospital Emergency Department, Iran

Elham Pishbin ${ }^{1}$, Shaghayegh Rahmani ${ }^{2}$

1. Emergency Medicine Department, Mashhad University of Medical Sciences, Mashhad/Iran

2. Imam Reza Hospital, Mashhad/Iran

Study/Objective: This project was aimed to evaluate waiting times in various processes in the $\mathrm{ED}$ of an academic hospital in Mashhad, Iran.

Background: Waiting for a physician visit in hospitals is one of the main factors associated with health care quality. Overcrowding is a significant problem in the Emergency Department (ED).

Methods: This cross sectional study was conducted in ED of our academic hospital in Mashhad, Iran. Data was gathered as a checklist. Intervals between triage and first visit, getting nursing care and discharge from ED were the main variables in our study. Data was entered using the SPSS version 16; P-value less than 0.05 was considered as significance level.

Results: In the first 25 days of the study, 1,250 patients were enrolled the study. Of those, 466 patients (37.2\%) were triaged in first and second level and 784 (62.8\%) were in third or fourth level. Mean duration between triage and first visit was $13.5 \pm 7.6$ minutes (first and second level of triage) and $16.4 \pm 10.1$ minutes (third and fourth level). Mean duration for receiving nursing care was $12.1 \pm 7.8$ minutes (first and second level of triage) and $15.6 \pm 9.5$ minutes (third and fourth level). There was a negative correlation between number of patients and waiting time for the first visit, in patients were in third and fourth level of triage $(\mathrm{p}<0.001, \mathrm{r}=0.654)$.

Conclusion: Our finding revealed that the number of ED physicians and nurses were correlated with waiting time for patients. So it seems, the use of strategies to reduce ED waiting time could be satisfying for both patients and staffs.

Prehosp Disaster Med 2017;32(Suppl. 1):s34

doi:10.1017/S1049023X17001066

\section{Increased Burdens of Emergency Departments -}

\section{Organizational Challenges}

Przemyslaw W. Gula ${ }^{1}$, Krzysztof Karwan ${ }^{2}$

1. Polish Armed Forces, Krakow/Poland

2. Polish Armed Forces, Warszawa/Poland

Study/Objective: The paper aims to assess Emergency Department (ED) organizational changes that are necessary due to constant increases of patient volume. We reviewed the ways of facilitating ED organization, and specifically the options of applying Lean analysis to optimize ED operations.
Background: The Military Institute of Medicine has one of the largest EDs in Poland, being a part of a multi-profile specialist hospital and regional trauma center for the Warsaw Agglomeration. The increase of the number of patients from 31,554 to 83,530, from 2009 to 2015 (a nationwide trend) was not matched by growth of the number of personnel or ED infrastructure, forcing change in work organization. The annual average of patients referred to the Trauma Center was 691.

Methods: Solutions to optimize ED operations were assessed in terms of the need to adapt to the growing ED burdens, specifically based on the results of application of the Lean analysis and the International Emergency Department Leadership Institute (IEDLI) standards.

Results: During the six years under review, ED's burdens increased by $264.7 \%$, but neither the infrastructure nor the number of personnel grew accordingly, necessitating a search for organizational solutions to keep up with patient flow and ensure patient safety. The number of patients not in life-threatening condition grew from $37 \%$ to $48 \%$ of $\mathrm{ED}$ patients, proving a major challenge. Continuity of operations requires patient flow modelling, triage system modification and efforts to decrease access block. Elements of Lean analysis and IEDLI recommendations were applied to keep safety standards for patients in life-threatening conditions, and Trauma Center patients (only $0.8 \%$ of ED patients but creating long-term burden).

Conclusion: The increase of the number of ED patients who have higher expectations and create greater burdens and duties for personnel and ED infrastructure, was not matched by an increase of the number of personnel and ED infrastructure development. Therefore, it is necessary to accept solutions to reduce risk and undesirable effects.

Prehosp Disaster Med 2017;32(Suppl. 1):s34

doi:10.1017/S1049023X17001078

\section{Risk Recognition: Rationing Emergency Department Care} as a Response to Overcrowding Sandra Richardson

Christchurch Hospital Emergency Department, Canterbury District Health Board, Christchurch/New Zealand

Study/Objective: The central objective of this research is to examine the relative risks associated with rationing Emergency Department (ED) care. A number of sub-objectives are considered, including: definition and measurement of overcrowding; rationale for managing so-called 'inappropriate' attendees; definitions of primary care patients; range of risks associated with rationing of care; acknowledgment and discussion of ethical issues resulting from these.

Background: Overcrowding and the associated impact on ED flow, patient outcomes and staff recruitment, retention and morale are increasingly recognized and reported. However, a core assumption remains that much of this is due to the presence of inappropriate attendees, or those who could be treated in primary care facilities. Efforts to address this include redirection of patients away from EDs, which effectively introduces rationing of care. This has not been made explicit, nor clearly discussed in the public arena, and the allocation of risk remains unequally distributed. 